\title{
Le Genre et la Sexualité dans le Roman 1'Amant de Marguerite Duras: Une Étude Selon Le Féminisme de Stevi Jackson
}

\section{Vina Fitriana ${ }^{\bowtie}$ Sunahrowi ${ }^{\bowtie}$ Ahmad Yulianto}

Département de la Langue et la Littérature Étrangère, Faculté des Langues et des Arts, Universitas Negeri Semarang, Indonesia

\begin{tabular}{|c|c|}
\hline Info d'article & Abstract \\
\hline $\begin{array}{l}\text { Histoire de l'Atrticle: } \\
\text { Reçu Janvier } 2020 \\
\text { Accepté Mars } 2020 \\
\text { Publié Mei } 2020\end{array}$ & Extrait \\
\hline $\begin{array}{l}\text { Keywords: } \\
\text { Le Féminisme, le genre et la } \\
\text { sexualité, L'Amant }\end{array}$ & $\begin{array}{l}\text { La littérature est divisée en deux, celle imaginative et celle non-imaginative. La littérature non- } \\
\text { imaginative se compose de l'essais, des critiques, des notes biographiques et des lettres, tandis que } \\
\text { la littérature imaginative se compose de la poésie, de la prose et du drame. Un exemple de la } \\
\text { littérature imaginative est le roman. Marguerite Duras est une femme écrivaine qui est née à Gia- } \\
\text { Dinh (L'Indochine-Française/ Viêt nam) en 1914. Ses œuvres sont beaucoup inspirés par sa propre } \\
\text { histoire de vie et son amour pour la littérature. Son roman intitulé L'Amant est publié par les } \\
\text { Éditions de Minuit en 1948, a reçu le prix Goncourt en 1986. Le roman français de Marguerite } \\
\text { Duras est un roman du style autobiographique. Ce roman raconte le voyage de l'amour de } \\
\text { l'écrivaine est très belle, très intime et plein de mystère. Le roman d'autobiogaphique est une } \\
\text { histoire d'amour intéressante. Ce type de roman est populaire dans cette époque. Ce roman a été } \\
\text { également traduit en Anglais. C'est pourqoui le chercheur a choisi ce roman comme object } \\
\text { matériel. Pour découvrir les faits de l'historique dans le roman L'Amant, le chercheur l'a examiné } \\
\text { en utilisant la théorie du féminisme de Stevi Jackson, sur le genre et la sexualité du personnage. } \\
\text { Cette recherce se concentre sur les faits historiques dans l'œuvre elle-même, donc cette étude } \\
\text { utilise une approche objective, afin d'exploiter autant que possible ces éléments, et en utilisant les } \\
\text { techniques de bibliographie pour l'acquisition de données comme source écrite. Le chercheur a } \\
\text { utilisé la methode analytique descriptive dans cette recherche. Le chercheur décrit les faits qui sont } \\
\text { suivi par analyse en utilisant des mots ordinaires, et non sous forme de nombres, de graphique, ou } \\
\text { de tableaux. Le chercheur a analysé comment le rôle du genre de personnage principale, la mère,le } \\
\text { frère et l'influence du genre sur le personnage principale ou le jeune fille, ensuite la sexsualité de } \\
\text { jeune fille et d'homme chinois. Le résultat d'analyse montre que le jeune fille a été influencé sur la } \\
\text { discussion du genre et de la sexualité. }\end{array}$ \\
\hline
\end{tabular}

(C) 2020 Universitas Negeri Semarang

\begin{tabular}{lc}
\hline Addresse: & ISSN 2252-6730 \\
Gedung B4 FBS Universitas Negeri Semarang & \\
Kampus Sekaran, Gunung pati, Semarang, 50229 &
\end{tabular}




\section{INTRODUCTION}

Une œuvre littéraire se compose des systèmes qui représentent des situations sociales. Un auteur est la réflexion de la condition socioculturelle. Les expériences de l'auteur ne se séparent jamais de son œuvre littéraire. On trouve une relation génétique entre l'auteur et la société qui réalisent la vision du monde (Faruk, 2012:25).

L'œuvre littéraire est partagée en trois grandes catégories, à savoir la prose, le théâtre, et la poésie. Chaque genre se forment de deux types, l'œuvre en écrit et l'œuvre en parole la poésie, la prose et drame en écrit est une résultat du procès créative dont l'auteur mets son idée en écrit ( Sukadaryanto, $2010: 1$ ).

L'œuvre littéraire est une expression personnelle de humain sous forme d'expérince, de pensée, de sentiment, d'idée, dans une image de la vie qui évoque le charme avec le langage et dépeint dans un écrit. Le roman est un belle l'œuvre littéraire. Le mot roman vient de la language française romance. Aupravant, le roman était plutôt l'œuvre littéraire ou toutes les lectures écrites en langue romance ou ancien française (Komaruddin, 2002: 222: 223).

Le roman L'Amant de Marguerite Duras a été publié en 1984 au Viêt nam à la fin de 1920. Le roman L'Amant de Marguerite Duras est un roman du style autobiographique qui raconte une fille qui est née et grandi à Gia-Dinh (L'Indochine-Française/ Viêt nam), comme on sait que L'Indochine a été Français colonie. Le personnage principal (la jeune fille) elle a quinze ans et elle vit en pensionnat. Au milieu de sa période d'adolescente, la jeune fille rencontre l'homme chinois riche et flamboyant, il a quarante ans. Leur histoire d'amour commence quand la jeune fille a montée la bord du ferry à traverse la rivière de Mekong et elle rencontre l'homme chinois qui était dans sa limousine.

Les œuvres littéraires caractérisées par le féminisme émergent comme une réaction à la domination masculine dans les œuvres littéraires. L'œuvre féministe apparaît comme un réaction de la domination des hommes dans l'œuvre littéraire. Il parait qu'on toujours étudie l'ouvre écrite par l'homme plus que celle écrite par la femme. Le féminisme est une branche du développement du monde littéraire avec la spécification des problèmes du genre. Le sens de genre elle-même est la distinction des hommes et des femmes selon la construction de la société faite par les humains et peut changer selon le lieu, le temps et la culture. Les femmes sont considérées qu'elles ont des sentiments, par exemple l'émotion, le tristesse. Elles se sacrifient à l'autre. Megawangi (1999:94).

\section{MÉTHODE DE RECHERCHE}

Pour l'approche de la recherche, j'utilise la théorie du féminisme de Stevyi Jackson. L'objet de cette recherche est de décrire le genre du personnage principal ou la jeune fille, la mère et le frère de personnage principal, l'influence du genre sur le comportement du personnage principale (la jeune fille), et la sexualité de personnage principale (la jeune fille et l'homme chinois), toujours selon cette théorie de Stevi Jackson.

Il y a deux sources des donnés dans cette recherche. Ce sont la source des données primaires et celle des données secondaires. La première source est le roman L'Amant, une œuvre de Marguerite Duras. Le deuxième est la théorie du féminisme de Stevi Jackson.

La méthode de collecte de données utilisée dans cette étude est la lecture attentive ainsi que la technique de notation. La méthode d'analyse des données est celle de la description analytique. Pour finir, la technique de l'analyse des données est celle de l'analyse contenue.

\section{RÉSULTAT ET DISCUSSION}

L'analyse se divise en plusieurs étapes : les rôles du genre et de la sexualité les personnages dans le roman L'Amant, le rôle du genre de la jeune fille, la mère et le frère, l'influences du genre sur 
le comportement du personnage principale, l'hétérosexuel de la jeune fille et l'homme chinois, le bisexuel de la jeune fille, et l'agressivité de la jeune fille.

\section{Le rôle du genre}

Le rôle du genre est le rôle que les hommes et les femmes jouent dans leurs droits et obligations. Par exemple comment une femme prend soigne et d'éduque d'enfant, et qu'un homme gagne de sa vie pour la famille.

Quinze ans et demi. C'est la traversée du fleuve. Quand je rentre à Saigon, je suis en voyage, surtout quand je prends le car. Et ce matin-là je pris le car à Sadec où ma mère dirige l'école des filles. C'est la fin des vacances scolaires, je ne sais plus lesquelles. Je suis allée le passer dans la petite maison de fonctionne ma mère. Et ce jour-là je reviens à Saigon, au pensionnat. Le car pour indigènes est partie de la place au du marché de Sadec. Comme d'habitude ma mère m'a accompagnée et elle m'a confiée au chauffeur, toujours elle me confie aux chauffeurs des cars de Saigon, pour le cas d'un accident, d'un incendie, d'un viol, d'un attaque de pirates (SET/1/6)

La citation <Et ce matin-là je pris le car à Sadec où ma mère dirige l'école des filles> décrit la mère de jeune fille qui a de rôle, de fonction et de responsabilité. Le rôle d'une mère devait prendre soin de la maison, mais elle soutient de sa famille. Elle a la responsabilité de s'occuper et de soutient sa famille parce que la mère est une veuve. Ensuit la citation < Comme d'habitude ma mère m'a accompagnée et elle m'a confiée au chauffeur, toujours elle me confie aux chauffeurs des cars de Saigon, pour le cas d'un accident, d'un incendie, d'un viol, d'un attaque de pirates $>$ explique la préoccupation d'une mère à sa fille, alors la mère a confié sa fille au le chauffeur de l'autobus par crainte de vol, de viol et d'autres. Les phrases explique que les femmes sont faibles, ne peuvent pas prendre soin d'elles-mêmes et ont une grande peur que les hommes. Cela fait référence au rôle du genre dans le personnage principal ou la jeune fille.

Faire que l'aîné soit tout à fait séparé des deux plus jeunes. Elle ne l'a pas fait. Elle a été imprudente, elle a été inconséquente, irresponsable. Elle était tout cela. Elle a vécu.( $\mathrm{SET} / 2 / 49)$.

La citation $<<$ Faire que l'aîné soit tout à fait séparé des deux plus jeunes. Elle ne l'a pas fait. Elle a été imprudente, elle a été inconséquente, irresponsable. Elle était tout cela. Elle a vécu>> décrit le rôle du genre le frère de la jeune fille comme un frère aîné devait prendre soin de son frère et de sa sœur et aime sa mère en aidant à faire de l'argent. Mais il compte vivre avec sa mère et n'est pas responsible.il n'est pas fait de ses responsibiltès en tant qu'homme.

\section{L'influence du genre sur le comportement de la jeune fille}

Comme le plus petit système social, la famille a une énorme influence en termes de caractère de construction d'un individu. La famille est le producteur et le consommateur, et doit préparer et fournir les nécessités quotidiennes comme les vêtements et les nourritures. L'enfance est un moment si important de pose les bases de la personnalité qui donnera de la couleur quand un enfant déviant un adulte. La qualité des modèles développement de l'enfance est très importante.

Maintenat je vois que très jeune à dix-huit ans, à quinze ans, j'ai eu ce visage prèmonitoire de celui que j'ai attrapé ensuite avel l'alcool dans l'âge moyende ma vie. L'alcool a rempli la fonction que Dieu n'a pas eue, il a eu aussi celle de me tuer, de tuer. Ce visage de l'alcool m'est venu avant l'alcool. L'alcool est venu le convirmer.( SET/3/5)

La citation ci-dessus explique comme un comportement du genre dans le personnage principale de l'adolescente, mais elle a un visage comme une personne beaucoup plus âgée que son 
âge parce que la jeune fille consommé souvent l'alcool, et sa triste histoire de vie. L'âge de jeune fille devait recevoir une orientation spirituelle de l'éducation morale de ses parents. En fait, la jeune fille ne reçoit pas plus d'attention de sa mère parce que sa mère est occupée à travailler.

\section{Le bisexuel chez la jeune fille}

Le bisexuel est des personnes qui s'intéressent aussi fortement aux homes et aux femmes de façon émotionnel, romantique, intellectuellement, et sexuel.

Je voudrais manger les seins d'Hèlène Lagonelle comme lui mange les seins de moi dans la chambre de la ville chinoise où je vais chaque soir approfondir la connaissance de Dieu. Etre dévorée de ces seins de fleur de farine que sont les siens. (SET 4/66)

Cette citation explique la jeune fille joue la scène comme un homme et une femme en dis "Je voudrais manger les seins d'Hèlène Lagonelle comme lui mange les seins de moi dans la chambre de la ville chinoise où je vais chaque soir". La jeune fille est très tentée par la beauté du corps d'Hélèle Lagonelle alors elle veut écouler sa désir sexuelle qui ne devrait pas être s'esprit parce qu'elle est aussi une femme.

\section{L'hétérosexuel de la jeune fille et l'homme chinois}

L'hétérosexuel est un l'intérêt sexuelle ou les habitudes sexuelles des personnes en sexe différent. En tant qu'orientation sexuelle se réfère à un modèle ou un caractère pour l'expérience de l'intérêt sexuel, l'affection physique contre des personnes de sexe différent.

Il devient brutal, son sentiment est désespéré, il se jette sur moi, il mange les seins d'enfant, il crie, il insulte. Je ferme les yeux sur le plaisir très fort. Je pense il a l'habitude, c'est ce qu'il fait dans la vie, l'amour, seulement ça. Les mains sont expertes, merveilleuses, parfaites. J'ai beaucoup de chance, c'est clair, c'est comme un métier qu'il aurait, sans le savoir il aurait le savoir exact de ce qu'il faut faire, de ce qu'il faut dire (SET/5/37).

Cette citation décrit l'hétérosexuel de la jeune fille et l'homme chinois parce qu'ils ont un intérêt sexuelle. Ils ont fait de rapport sexuel comme un couple marié.

\section{L'agressivité chez la jeune fille}

L'agressivité est une forme de comportement qui appartient à tout le monde. Freud a dit que les humains ont des impulsions ou des instincts innés pour se battre. Comme l'expérience physiologique de la faim, de la soif ou de la montée de poussée sexuelle, alors il est prouvé que les humains ont un instinct inné de se comporter agressivement.

Elle lui dit qu'elle ne veut pas qu'il lui parle, que ce qu'elle veut c'est qu'il fasse comme d'habitude il fait avec les femmes qu'il emmène dans sa garçonnière. Elle le supplie de faire de cette façon-là. Il a arraché la robe, il la jette, il a arraché le petit slip de coton blanc et il la porte ainsi nue jusqu'au lit. Et alors il se tourne de l'autre côté du lit et il pleure. Et elle, lente, patiente, elle le ramène vers elle et elle commence à le déshabiller. Les yeux fermés, elle le fait. Lentement. Il veut faire des gestes pour l'aider. Elle lui demande de ne pas bouger. Laisse-moi. Elle dit qu'elle veut le faire elle. Elle le fait. Elle le déshabille. Quand elle le lui demande il déplace son corps dans le lit, mais à peine, avec légèreté, comme pour ne pas la réveiller. (SET /6/31-22)

Cette citation montre l'agressivité de la jeune fille, quand ils font la conversation, elle lui interrompt et lui demande de l'acte sexuelle avec elle come il l'a fait avec d'autres femmes. La jeune fille qui a commencé à le faire, elle qui a dépouillé les vêtements de l'homme. La citation décrit si la poussée sexuelle de la jeune fille est plus grande que l'homme chinois. 


\section{CONCLUSION}

Chaque individu a une faiblesse et un force. Dieu créé un homme et une femme afin qu'ils se complètent, mais en généralement les gens supposent que les femmes sont plus faibles et plus inférieures que les hommes. Les hommes sont considérés comme plus forts et plus courageux, et les femmes sont considérées peureuses, faciles à pleurer et ne peuvent pas garder soin d'elles-mêmes. Bien que les femmes soient des créatures faibles, mais beaucoup de femmes peuvent faire le travail des hommes, par exemple le personnage qui joue la mère dans le roman L'Amant, la mère peut jouer son rôle de mère et de père pour ses enfants parce qu'elle est une veuve alors elle travaille pour répondre aux besoins de sa famille et prend soigne et d'éduque ses enfants. Le caractère d'homme Chinois dans le roman L'Amant prouve qu'ne femme peut exprimer son amour sans plus attendre un homme pour l'exprimer à l'avance.

\section{BIBLIOGRAPHIE}

